University of Nebraska - Lincoln

DigitalCommons@University of Nebraska - Lincoln

\title{
Temperature Dependence of the Surface Anisotropy of Fe Ultrathin Films on $\mathrm{Cu}(001)$
}

\author{
Axel Enders \\ University of Nebraska at Lincoln, a.enders@me.com \\ D. Peterka \\ Max-Planck-Institut für Festkörperforschung \\ D. Repetto \\ Max-Planck-Institut für Festkörperforschung \\ N. Lin \\ Max-Planck-Institut für Festkörperforschung \\ Ivan Dmitriev \\ Max-Planck-Institut für Festkörperforschung, dmitriev@fkf.mpg.de \\ See next page for additional authors
}

Follow this and additional works at: https://digitalcommons.unl.edu/physicsenders

Part of the Physics Commons

Enders, Axel; Peterka, D.; Repetto, D.; Lin, N.; Dmitriev, Ivan; and Kern, Klaus, "Temperature Dependence of the Surface Anisotropy of Fe Ultrathin Films on $\mathrm{Cu}(001)$ " (2003). Axel Enders Publications. 20.

https://digitalcommons.unl.edu/physicsenders/20

This Article is brought to you for free and open access by the Research Papers in Physics and Astronomy at DigitalCommons@University of Nebraska - Lincoln. It has been accepted for inclusion in Axel Enders Publications by an authorized administrator of DigitalCommons@University of Nebraska - Lincoln. 


\section{Authors}

Axel Enders, D. Peterka, D. Repetto, N. Lin, Ivan Dmitriev, and Klaus Kern 


\title{
Temperature Dependence of the Surface Anisotropy of Fe Ultrathin Films on $\mathrm{Cu}(001)$
}

\author{
A. Enders, ${ }^{*}$ D. Peterka, D. Repetto, N. Lin, A. Dmitriev, and K. Kern \\ Max Planck Institute for Solid State Research, Heisenbergstrasse 1, 70569 Stuttgart, Germany
}

(Received 18 December 2002; published 30 May 2003)

\begin{abstract}
We report an experimental approach to separate temperature dependent reversible and irreversible contributions to the perpendicular magnetic anisotropy of Fe films grown at low temperatures on $\mathrm{Cu}(001)$ substrates. The surface anisotropy $K_{S}(T)$ is found to decrease linearly with temperature, causing a thermally induced spin reorientation into the plane. The irreversible shift of the spin reorientation transition and the coercivity of the iron films are directly correlated to the increasing Fe island size during annealing. The increased coercivity is discussed in terms of domain wall energy inhomogeneities provided by the islands.
\end{abstract}

DOI: 10.1103/PhysRevLett.90.217203

It has been demonstrated for $\mathrm{Fe}$ films on $\mathrm{Cu}$ that the easy axis of magnetization can be switched by changing the film temperature [1-3]. The observed phenomenon is ascribed to temperature dependent magnetic anisotropy constants. A direct determination of the magnetic surface anisotropy $K_{S}(T)$, as done for a few other systems [4-6], has not been made. The lack of experimental data in this field hinders the development of more general theoretical models about the temperature dependence of the surface anisotropy. This is mainly due to the experimental difficulty to separate pristine magnetic effects from thermally induced structural changes. Irreversible changes, such as the change in roughness or intermixing at the interface, are known to alter the anisotropy significantly [7] and superimpose the reversible contribution, making independent measurements difficult. However, thermally induced structural driven changes of the magnetic film properties offer the chance to study the basic relation between magnetism and film structure.

In this Letter, we present an experimental approach to separate temperature dependent reversible and irreversible contributions to the magnetic anisotropy of epitaxial Fe films on $\mathrm{Cu}(001)$. Thus, both contributions can be determined independently. We find a reversible linear decrease of the surface anisotropy constant $K_{S}(T)$ in the temperature range between 127 and $300 \mathrm{~K}$. On the other hand, we exploit temperature induced irreversible changes of the spin reorientation transition (SRT) and the coercivity $H_{C}$ to quantify the correlation between microstructure and magnetism.

As a model system, we study epitaxial fcc-Fe films grown at $120 \mathrm{~K}$ on $\mathrm{Cu}(001)$. The structure and magnetism of the films is known to strongly deviate from Fe bulk behavior. Below a thickness of $t_{\mathrm{Fe}} \sim 4.3 \mathrm{ML}$, a tetragonally distorted fcc structure is found [8,9]. In the same thickness region, the easy axis of magnetization is reoriented out of the film plane [10], ascribed to dominating surface anisotropy contributions $[11,12]$. The morphology sensitively depends on the growth temperature [13] and the thermal treatment after growth [14]. We focus on low
PACS numbers: 75.30.Gw, 75.60.Ej, 75.60.Nt, 75.70.Ak

temperature grown films since they exhibit enhanced surface roughness compared to films grown at room temperature $[13,15]$. Thermally activated changes of the film morphology and its consequences for the film magnetism can thus ideally be studied.

The films have been grown at substrate temperatures of $120 \mathrm{~K}$ in a UHV system with a base pressure of $7 \times$ $10^{-11}$ mbar. Fe films with varying thickness have been produced by depositing over a straight metal plate mounted on the front end of the evaporator, thus producing a half shadow region of deposition on the sample surface. Magnetic investigations have been done by in situ Kerr microscopy as well as standard magnetooptical Kerr-effect measurements [16]. The critical thickness $t_{\text {crit }}$ for spin reorientation in ultrathin $\mathrm{Fe}$ films on $\mathrm{Cu}(100)$ is governed mainly by the magnetic interface anisotropy arising at the film-vacuum and -substrate interfaces, $K_{\mathrm{f}-\mathrm{vac}}$ and $K_{\mathrm{f}-\mathrm{s}}[12]$,

$$
t_{\text {crit }}=\frac{2\left(K_{\mathrm{f}-\mathrm{vac}}+K_{\mathrm{f}-\mathrm{s}}\right)}{2 B_{1}\left(\varepsilon_{\perp}-\varepsilon_{\|}\right)+\mu_{0} M_{S}^{2}} .
$$

Here $B_{1}$ is the bulk magnetoelastic (ME) coupling constant, $\varepsilon_{\perp}$ and $\varepsilon_{\|}$being the in-plane and out-of-plane strain, and $M_{S}$ is the saturation magnetization. The observed critical thickness of the as-grown film, $t_{\text {crit }}=$ 4.3 ML, is reproduced by inserting $K_{\mathrm{f}-\mathrm{vac}}=$ $0.89 \mathrm{~mJ} / \mathrm{m}^{2}, K_{\mathrm{f}-\mathrm{s}}=0.6 \mathrm{~mJ} / \mathrm{m}^{2}, M_{S}=1751 \mathrm{kA} / \mathrm{m}, \varepsilon_{\|}=$ $5 \%$, and $\varepsilon_{\perp}=1.54 \%$ ([12] and references therein). An effective $B_{1}^{\text {eff }}\left(\varepsilon_{\|}\right)=13.5 \mathrm{MJ} / \mathrm{m}^{3}$ has been used to account for the strain dependence of the ME coupling [17]. Assuming the effective epitaxial strain and the $B_{1}$ to be independent of the film temperature, as it is for bulk below $300 \mathrm{~K}$ [18], changes in $t_{\text {crit }}$ are attributed solely to a temperature dependence of the magnetic surface anisotropy. This approximation is based on the fact that the surface anisotropy is by far the dominant energy contribution, and changes in $B_{1}^{\text {eff }}$ during the growth of already existing islands are small. 
Temperature driven reversible and irreversible changes of the magnetic properties of $\mathrm{Fe}$ on $\mathrm{Cu}$ can separately be identified by monitoring the magnetism during annealing/cooling cycles. Such an experiment is shown in Fig. 1. The Kerr image in Fig. 1(a) shows the remanent magnetization of a Fe wedge around the SRT. The change in the grey level at position $\alpha$ marks the critical thickness at which the easy axis of magnetization switches from perpendicular (dark) to in-plane (bright). Figure 1(b) shows the shift of the SRT towards a smaller thickness $\beta=3.73 \mathrm{ML}$ during heating of the sample up to $280 \mathrm{~K}$ with a rate of approximately $5-10 \mathrm{~K} / \mathrm{min}$. No further shift is observed when the sample temperature is held constant at the annealing temperature $T_{a}$ for $60 \mathrm{~min}$. Subsequent cooling back to $127 \mathrm{~K}$ also shifts the SRT back to a higher thickness $\gamma \neq \alpha$ [Fig. 1(c)], but the critical thickness as in Fig. 1(a) cannot be achieved. Repeating the experiment now shifts the SRT reversibly between $\beta$ and $\gamma$. Thus, at a given Fe thickness, the easy axis of magnetization can be switched reversibly between out-of-plane and in-plane by changing the temperature.

The full temperature dependence of $t_{\text {crit }}$ between 127 and $300 \mathrm{~K}$ is shown in Fig. 2(a). During the experiment the sample temperature was cycled between $127 \mathrm{~K}$ and $T_{a}$, as already shown in Fig. 1. In every subsequent warmingcooling cycle, the $T_{a}$ was increased by approximately $20 \mathrm{~K}$ with respect to the previous cycle, until $T_{a}=300 \mathrm{~K}$ was reached. The $t_{\text {crit }}$ was recorded at each temperature after realignment of the spins by an external field pulse of $H=$ $25 \mathrm{mT}$. The solid dots (O) in Fig. 2(a) represent the critical thickness at the indicated annealing temperature. Plotted at the same temperature as open dots $(O)$ is the changed critical thickness after the sample was being cooled back to $127 \mathrm{~K}$ from the respective $T_{a}$. Thus, these data are taken at identical experimental conditions, i.e., the same temperature. They therefore reflect the irrevers-
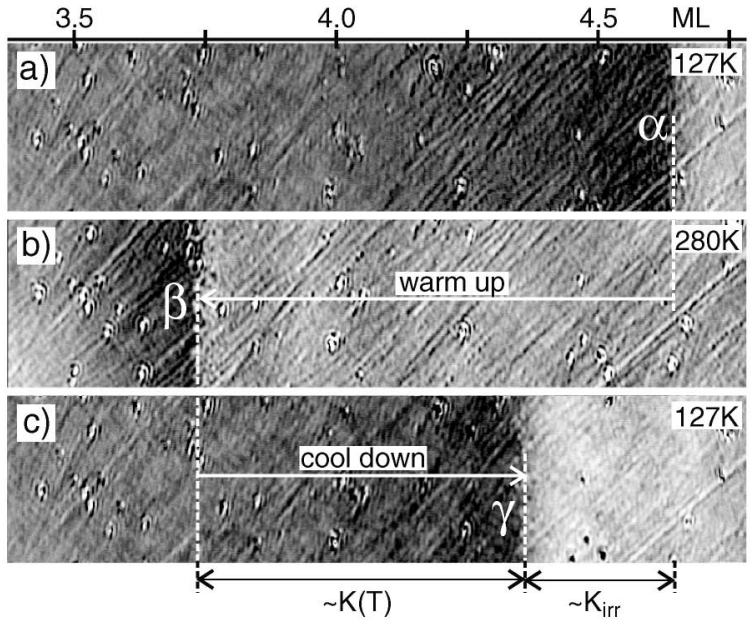

FIG. 1. Thermally induced shift of the reorientation transition of a Fe wedge. (a),(b) $t_{\text {crit }}$ changes from $\alpha$ to $\beta$ when raising the temperature from 127 to $280 \mathrm{~K}$. (c) Cooling back to $127 \mathrm{~K}$ shifts the reorientation transition to $\gamma \neq \alpha$. ible changes of the critical thickness after the annealing. In contrast, the difference between the two data sets in Fig. 2(a) represents purely the reversible shift of the critical thickness with temperature, analogous to the shift of $t_{\text {crit }}$ between $\beta$ and $\gamma$ in Fig. 1. Subtracting both data sets in Fig. 2(a) yields the linear dependence depicted in Fig. 2(b).

We ascribe the reversible change in $t_{\text {crit }}$ to the temperature dependence of the surface anisotropy constants of the film, $K_{S}=K_{\mathrm{f}-\mathrm{vac}}+K_{\mathrm{f}-\mathrm{s}}$. The irreversible changes are attributed to thermally activated structural changes in the film. In this picture, $K_{S}$ as the origin of the perpendicular magnetization may be separated into a temperature dependent reversible and an irreversible contribution,

$$
K_{S}=K(T)+K_{\text {irr }}
$$

In this notation, $K(T)$ is the anisotropy of a film that is assumed not to change its structure during annealing. By performing the experiment shown in Fig. 1, both contributions, $K(T)$ and $K_{\text {irr }}$, could be separated experimentally for ultrathin films for the first time. The trick used here is to cycle the temperature between annealing and growth temperature. After the structural changes are fully accomplished during the first cycle, $K_{\text {irr }}$ is zero in the subsequent cycle. Thus, the reversible shift with temperature can exclusively be recorded. The difference of $t_{\text {crit }}$ between $127 \mathrm{~K}$ and $T_{a}$ is plotted in Fig. 2(b) and converted into $K(T)$ using expression (1). We find $K(T)$ to decrease linearly with respect to its value at $T=127 \mathrm{~K}$ as the temperature is increased in the range below $300 \mathrm{~K}$.

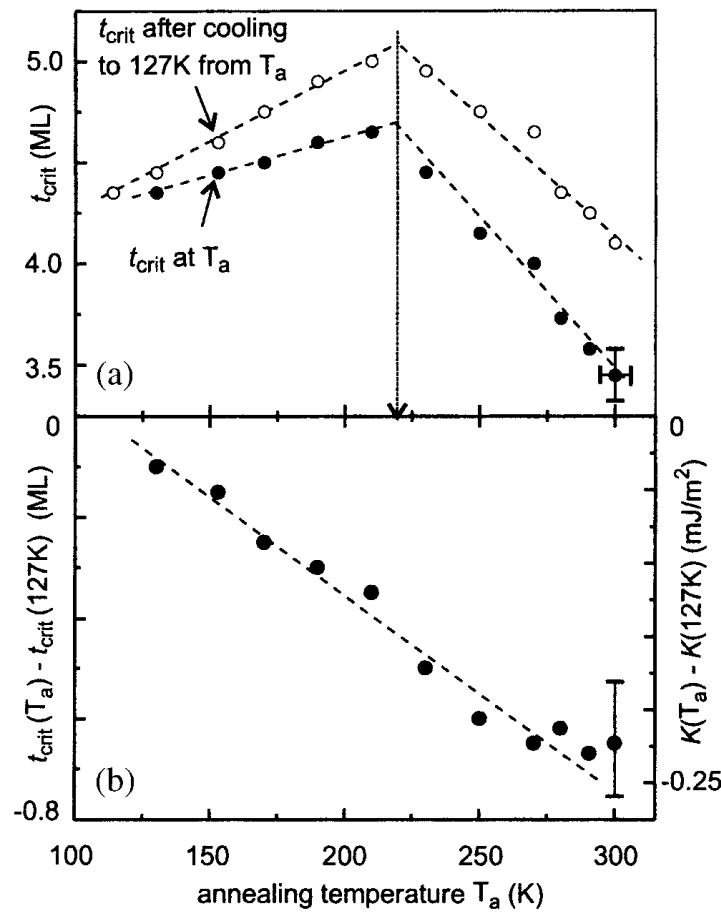

FIG. 2. (a) Temperature dependence of the critical thickness in the temperature range between $127 \mathrm{~K}$ and RT. (b) The difference of both data sets in (a) is proportional to $K(T)$. 
Qualitatively, the result agrees with theoretical models that predict a reduced anisotropy as the thermally induced jiggling of the spins reduces the expectation value of the magnetization. It can be shown that the anisotropy $K(T, H)$ follows the temperature dependence of the reduced saturation magnetization, $[M(T, H) / M(0)]^{b}$, and therefore vanishes at the Curie temperature $T_{C}$ [19]. Calculations of the anisotropy coefficients of a ferromagnetic Heisenberg monolayer also reflect this tendency, independent of the underlying method [20]. A quantitative comparison of our data with such models requires an analysis in terms of $T / T_{C}$. For the films investigated, $T_{C}$ is well above $300 \mathrm{~K}$ and could therefore not be measured due to the onset of intermixing at the $\mathrm{Fe} / \mathrm{Cu}$ interface. However, similar results of decreasing surface anisotropy with temperature have been found by other authors for $\mathrm{Ni} / \operatorname{Re}(0001)$ [5] and $\mathrm{Ni} / \mathrm{W}(110)$ [6]. The trend of the few data available suggests that the destruction of the perpendicular ferromagnetic ordering of the spins by the thermal agitation at the Curie temperature is always preceded by a spin reorientation into the plane.

The data in Fig. 2(a) clearly reflect the competition of anisotropy contributions of opposite sign. While annealing of the films below $220 \mathrm{~K}$ causes an increase of $t_{\text {crit }}$, further annealing above $220 \mathrm{~K}$ reduces it. In the following, we discuss the observed temperature dependence of $t_{\text {crit }}$ in Fig. 2(a) in terms of a competition between thermally activated morphological changes, superimposed by the reversible $K_{S}(T)$ dependence in Fig. 2(a). The morphology changes in the surface structure of a three monolayer thin $\mathrm{Fe}$ film grown at $120 \mathrm{~K}$ on $\mathrm{Cu}(001)$ have been studied during annealing by variable temperature scanning tunneling microscopy (STM). Figures 3(a)-3(d) show STM images of the film at four different temperatures. The following conclusions can be drawn from these images: (i) Below $\sim 240 \mathrm{~K}$ the island size increases from $\leq 1 \mathrm{~nm}$ up to $\approx 5 \mathrm{~nm}$ with temperature. (ii) No further island size growth is observed above $240 \mathrm{~K}$ up to room temperature. (iii) The rms roughness of the film of $\sigma=$ $1.5 \pm 0.5 \AA$ remains unchanged in the temperature range investigated. In other words, visible improvements of the film quality mainly affect the island diameter and are accomplished below $240 \mathrm{~K}$.

Based on this result, we conclude that the observed increase of $t_{\text {crit }}$ below $220 \mathrm{~K}$ in Fig. 2(a) is a consequence of the enlargement of the island diameter in the film. This is in full agreement with arguments given by Bruno, according to which the magnetic surface anisotropy $K_{S}$
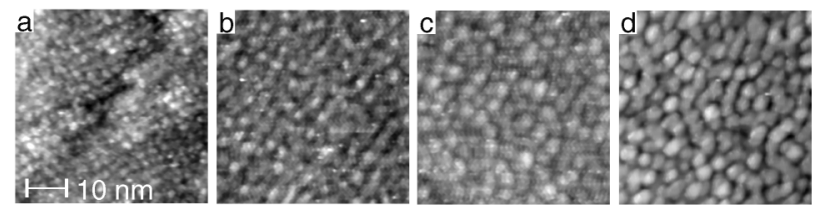

FIG. 3. STM images of a $3 \mathrm{ML}$ Fe film grown on $\mathrm{Cu}(100)$ at $120 \mathrm{~K}$, (a) at 165 , (b) 215 , (c) 238 , and (d) $296 \mathrm{~K}$. of a film with surface roughness $\sigma$ and island width $L$ is reduced by $\Delta K_{S}$ with respect to the ideally flat surface, following the expression $\Delta K_{S} / K_{S}=-2 \sigma / L$ [7]. For the Fe films studied here, this means that, although the $\sigma$ remains unchanged, the surface anisotropy in the asgrown film is smaller than in the annealed film due to the smaller island size $L$. The $K_{S}$ increases with $T$ due to the increase of $L$ shifting the $t_{\text {crit }}$ towards higher thickness. This effect is opposed by the reversible temperature dependence of $K_{S}(T)$, which tends to decrease $t_{\text {crit }}$ in the whole temperature range investigated.

After the island formation is accomplished at $240 \mathrm{~K}$, another effect irreversibly decreasing $t_{\text {crit }}$ becomes visible at higher temperatures in Fig. 2(a). One might speculate whether the formation of bcc crystallites during warming, as have been seen with high resolution STM on RT grown Fe films [21], reduce the perpendicular anisotropy by gradually making the fcc film more bcclike. This idea is supported by recent experiments showing drastic changes of the morphology of a RT grown fcc-Fe film on $\mathrm{Cu}(001)$ after cooling and subsequent warming up to $300 \mathrm{~K}$, ascribed to a transition of the film towards bcc structure [14]. Thermally activated interdiffusion between $\mathrm{Fe}$ and $\mathrm{Cu}$, which is known to alter the magnetic anisotropy energy [22], does not have to be considered here since it is found to be negligible below $300 \mathrm{~K}$ [23].

Also, the coercivity $H_{C}$ is significantly increased by the island growth. This becomes evident from $H_{C}(T)$ measurements during annealing of $3 \mathrm{ML} F e$ grown at $120 \mathrm{~K}$ (Fig. 4). The temperature dependence of $H_{C}$ during the first annealing directly after growth $(+)$ looks markedly different than during subsequent annealing where the $H_{C}(T)$ reproducibly follows the curve represented by (O). Clearly, after the first annealing the $H_{C}$ at $127 \mathrm{~K}$ is irreversibly increased and decays exponentially with temperature (dashed line).

The observed enhancement of $H_{C}$ can be explained within a model of energy inhomogeneities due to

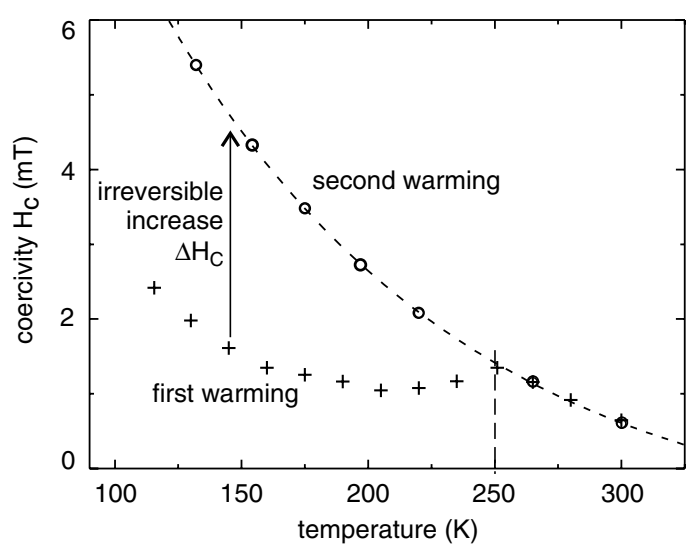

FIG. 4. Temperature dependence of the coercivity of 3 ML Fe grown at $127 \mathrm{~K}$ during the first $(+)$ and subsequent $(\bigcirc)$ annealing. 


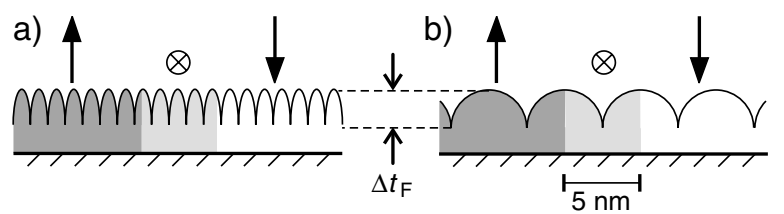

FIG. 5. Model of the Bloch domain wall (BW) width with respect to the island size. Annealing of the as-grown film (a) results in island sizes comparable to the BW width (b).

the film thickness variation $\Delta t_{F}$, taking into account the increase of the Fe island size. The energy and, thus, the field needed to reverse the magnetization in the sample are determined by the variation of the free energy of the domains, $H_{C} \sim \partial E / \partial r$. The enhanced domain wall energy at the island sites provides the main obstacle for domain wall propagation, thus determining the coercivity. The domain wall energy varies with film thickness due to exchange and anisotropy inhomogeneities [24]. The wall exchange energy variation is deduced from the morphology data in Fig. 3 and found to be $\Delta \gamma=1 \times$ $10^{-11} \mathrm{~J} / \mathrm{m}$ as a result of $\Delta t_{F}=4.8 \AA$. Additionally, the magnetic anisotropy due to thickness effects and strain relaxation varies with island size, further contributing to the energy barriers at the island sites. This means that it is less favorable for the domain wall to enter the 3D islands due to the enhanced wall energy there. Domain wall movement is most efficiently blocked if the island size $L$ becomes comparable to the Bloch wall width $\delta=\sqrt{A t_{F} / K_{S}}$.

Inserting the film thickness of $3 \mathrm{ML} \mathrm{Fe}, t_{F}=5.4 \AA$, exchange stiffness $A=2 \times 10^{-11} \mathrm{~J} / \mathrm{m}$, and $K_{S}=$ $1.49 \mathrm{~mJ} / \mathrm{m}^{2}$ gives the wall width of $\delta=6 \mathrm{~nm}$. This reveals the origin of the increase of $H_{C}$, as illustrated in Fig. 5: Without annealing, the domain wall stretches over several islands [Fig. 5(a)]. Wall movement is easy in this case. With increasing film temperature, the islands become bigger and reach at $T \sim 240 \mathrm{~K}$ a size comparable to the domain wall width, $L \cong \delta$. Now the wall is being trapped between two adjacent energy barriers. Further increase of the temperature above $240 \mathrm{~K}$ does not further change the island size, and the change of $H_{C}$ with temperature is fully reversible $\left(\Delta H_{C}=0\right)$. Repeating the experiment gives reproducibly the exponential dependence of $H_{C}(T)(\bigcirc)$ simply due to thermally activated overcoming of the energy barriers at the island sites. Although the islands themselves provide these energy barriers, they do not represent the Barkhausen volume. In temperature dependent magnetic viscosity measurements, we found the Barkhausen volume to vary from $80 \mathrm{~nm}$ at $127 \mathrm{~K}$ up to $220 \mathrm{~nm}$ at RT, thus being much larger than the Fe islands. These results are in full agreement with similar experiments published in Ref. [25].

In conclusion, reversible and irreversible thermal contributions to the magnetism of epitaxial Fe films have been separated experimentally, allowing for their individual investigation. We find that the surface anisotropy decreases reversibly with increasing temperature, causing a spin reorientation into the film plane. The irreversible change of the critical thickness and the coercivity with temperature could clearly be correlated to the change of the island size.

*Electronic addresses: a.enders@fkf.mpg.de

[1] D. P. Pappas, K.-P. Kämper, B. P. Miller, H. Hopster, D. E. Fowler, A. C. Luntz, C. R. Brundle, and Z.-X. Shen, J. Appl. Phys. 69, 5209 (1991).

[2] R. Allenspach and A. Bischof, Phys. Rev. Lett. 69, 3385 (1992).

[3] R. Allenspach, J. Magn. Magn. Mater. 129, 160 (1994).

[4] G. Garreau, E. Beaurepaire, K. Ounadjela, and M. Farle, Phys. Rev. B 53, 1083 (1996).

[5] R. Bergholz and U. Gradmann, J. Magn. Magn. Mater. 45, 389 (1984).

[6] M. Farle, B. Mirwald-Schulz, A. Anisimov, W. Platow, and K. Baberschke, Phys. Rev. B 55, 3708 (1997).

[7] P. Bruno, J. Phys. F: Met. Phys. 18, 1291 (1988).

[8] H. Zillgen, B. Feldmann, and M. Wuttig, Surf. Sci. 321, 32 (1994).

[9] S. Müller, P. Bayer, A. Kinne, C. Reischl, R. Metzler, and K. Heinz, Surf. Sci. 322, 21 (1995).

[10] S. Müller, P. Bayer, C. Reischl, K. Heinz, B. Feldmann, H. Zillgen, and M. Wuttig, Phys. Rev. Lett. 74, 765 (1995).

[11] J. Shen, A. K. Swan, and J. F. Wendelken, Appl. Phys. Lett. 75, 2987 (1999).

[12] D. Peterka, A. Enders, G. Haas, and K. Kern, Phys. Rev. B 66, 104411 (2002).

[13] J. Giergiel, J. Shen, J. Woltersdorf, A. Kirilyuk, and J. Kirschner, Phys. Rev. B 52, 8528 (1995).

[14] D. Qian, X. F. Jin, J. Barthel, M. Klaua, and J. Kirschner, Phys. Rev. Lett. 87, 227204 (2001).

[15] E. Mentz, D. Weiss, J. Ortega, A. Bauer, and G. Kaindl, J. Appl. Phys. 82, 482 (1997).

[16] D. Peterka, A. Enders, G. Haas, and K. Kern, Rev. Sci. Instrum. 74, 2744 (2003).

[17] T. Gutjahr-Löser, Ph.D. thesis, Martin-LutherUniversität Halle Wittenberg, 1999.

[18] M. B. Stearns, in Magnetic Properties of Metals, edited by O. Madelung and H. P. J. Wijn, Landolt-Börnstein, New Series, Group III, Vol. 19, Pt. a (Springer-Verlag, Berlin, 1986), p. 49.

[19] E. Callen, J. Appl. Phys. 53, 8139 (1982).

[20] A. Ecker, P. Fröbrich, P. Jensen, and P. Kuntz, J. Phys. Condens. Matter 11, 1557 (1999).

[21] A. Biedermann, R. Tscheliessnig, M. Schmid, and P. Varga, Phys. Rev. Lett. 87, 086103 (2001).

[22] B. Úfalussy, L. Szunyogh, and P. Weinberger, Phys. Rev. B 54, 9883 (1996).

[23] D. Steigerwald, I. Jacob, and J.W. F. Egelhoff, Surf. Sci. 202, 472 (1988).

[24] D. Sander, R. Skomski, C. Schmidthals, A. Enders, and J. Kirschner, Phys. Rev. Lett. 77, 2566 (1996).

[25] A. Kirilyuk, J. Giergiel, J. Shen, and J. Kirschner, J. Magn. Magn. Mater. 159, L27 (1996). 